\title{
A Table of Generalized Circular Error
}

\author{
By Harry Weingarten and A. R. Di Donato
}

1. Introduction. This note provides an abbreviated table (Table 1) giving solutions for the value of $K$ satisfying

$$
\frac{1}{2 \pi \sigma_{x} \sigma_{y}} \int_{R} \int \exp \left[-\frac{1}{2}\left(\frac{x^{2}}{\sigma_{x}^{2}}+\frac{y^{2}}{\sigma_{y}^{2}}\right)\right] d x d y=P
$$

where $R$ is the circle $x^{2}+y^{2}=K^{2} \sigma_{x}^{2}, \sigma_{x} \geqq \sigma_{y}$ and $c=\sigma_{y} / \sigma_{x} . K$ has been computed for $c=0(.01) 1$ and $P=0(.01) .99$. The table provided here will not contain all the results because of space limitations. The complete table is available upon request directed to either author. It differs from the extensive one in [1] which also gives numerous applications and a wide bibliography of the bivariate normal distribution.

2. Application. When $P=.5$ and $c=1$ we obtain the cPE (circular probable error) relationship used in ballistic studies. In this case $K=1.17741$, which may easily be found without the table in this note. When $c \neq 1$, however, (which is the usual case) it is still of interest to find the circles within which impacts will occur with given probabilities, rather than the ellipses. For any particular $P$ and $c$ the value of $K$ in the table multiplied by $\sigma_{x}$ is the required radius.

3. Statistical Interpretation. This kind of problem has been widely considered as indicated by the references in [2], where the approach is differently oriented, being concerned with the general problem of the distribution of quadratic forms. Essentially we consider here the cumulative distribution in tabular form of the random variable,

$$
Z=X^{2}+Y^{2}
$$

where $X$ and $Y$ are independently and normally distributed with zero means and variances $\sigma_{z}$ and $\sigma_{y}$. ( $Z$ does not, of course, have a $\chi^{2}$ distribution unless $\sigma_{x}=\sigma_{y}=$ 1.) In [3], Chapter 27, there will be found application of such results to the specification of regions of type $C$ in the testing of hypotheses.

4. Analysis. This section will detail the computational and numerical analysis aspects of the preparation of the table.

The probability integral under consideration is given by the following equation:

$$
P\left(K, \sigma_{x}, \sigma_{y}\right)=\frac{1}{2 \pi \sigma_{x} \sigma_{y}} \int_{R} \int \exp \left[-\frac{1}{2}\left(\frac{x^{2}}{\sigma_{x}^{2}}+\frac{y^{2}}{\sigma_{y}^{2}}\right)\right] d x d y
$$

where the region, $R$, is specified as a circle with its center at the origin and with radius $K \sigma_{x}$. The use of polar coordinates transforms (1) to

$$
P(K, c)=\frac{1}{2 \pi c} \int_{0}^{2 \pi} \int_{0}^{\pi} \exp \left[-\frac{1}{2} \rho^{2}\left(\frac{1+c^{2}}{2 c^{2}}-\frac{1-c^{2}}{2 c^{2}} \cos 2 \theta\right)\right] \rho d \rho d \theta
$$

Received June 27, 1960. 
TABLE 1

The Generalized Circular Probable Error $K$

\begin{tabular}{|c|c|c|c|c|c|c|c|c|c|c|}
\hline & .05 & .10 & 15 & .20 & .25 & .30 & .35 & .40 & .45 & .50 \\
\hline $\begin{array}{l}.05 \\
.10 \\
.15 \\
.20 \\
.25 \\
.30 \\
.35 \\
.40 \\
.45\end{array}$ & $\begin{array}{l}0.08149 \\
0.13631 \\
0.19590 \\
0.25834 \\
0.32259 \\
0.38558 \\
0.55653 \\
0.52479 \\
0.59956 \\
0.67635\end{array}$ & $\begin{array}{l}0.10697 \\
0.1632 \\
0.21757 \\
0.2754 \\
0.33506 \\
0.39867 \\
0.46500 \\
0.5309 \\
0.60623 \\
0.68199\end{array}$ & $\begin{array}{l}0.12806 \\
0.19017 \\
0.24565 \\
0.30048 \\
0.35715 \\
0.41685 \\
0.48004 \\
0.54679 \\
0.61721 \\
0.69163\end{array}$ & $\begin{array}{l}0.14627 \\
0.2149 \\
0.27316 \\
0.32894 \\
0.38470 \\
0.44210 \\
0.50225 \\
0.56592 \\
0.63363 \\
0.70585\end{array}$ & $\begin{array}{l}0.16251 \\
0.22662 \\
0.29897 \\
0.35690 \\
0.41348 \\
0.47050 \\
0.52924 \\
0.59073 \\
0.65585 \\
0.72543\end{array}$ & $\mid$\begin{tabular}{l|}
0.17730 \\
0.25701 \\
0.32313 \\
0.38367 \\
0.44188 \\
0.49965 \\
0.55829 \\
0.61889 \\
0.68244 \\
0.74994
\end{tabular} & $\begin{array}{l}0.19097 \\
0.27599 \\
0.34585 \\
0.40917 \\
0.46941 \\
0.52853 \\
0.58788 \\
0.64854 \\
0.71154 \\
0.77788\end{array}$ & $\begin{array}{l}.20375 \\
.29383 \\
.36734 \\
.43349 \\
.49596 \\
.55677 \\
.61731 \\
.67866 \\
.74184 \\
.80785\end{array}$ & \begin{tabular}{|l|}
0.21579 \\
0.31070 \\
0.38777 \\
0.45676 \\
0.52155 \\
0.58424 \\
0.64626 \\
0.70872 \\
0.77260 \\
0.83890
\end{tabular} & $\begin{array}{l}.22721 \\
.32675 \\
.40729\end{array}$ \\
\hline $\begin{array}{l}.55 \\
.60 \\
.65 \\
.70 \\
.75 \\
.80 \\
.85 \\
.90\end{array}$ & $\begin{array}{l}0.75707 \\
0.84311 \\
0.93593 \\
1.03764 \\
1.15144 \\
1.28253 \\
1.440+0 \\
1.64561 \\
1.96060\end{array}$ & $\begin{array}{l}0.76210 \\
0.84761 \\
0.93998 \\
1.04129 \\
1.15+73 \\
1.28548 \\
1.44303 \\
1.64791 \\
1.96253\end{array}$ & $\begin{array}{l}0.77066 \\
0.85527 \\
0.94685 \\
1.04748 \\
1.16029 \\
1.29046 \\
1.44746 \\
1.65179 \\
1.96578\end{array}$ & $\begin{array}{l}0.78314 \\
0.86634 \\
0.95675 \\
1.05635 \\
1.16825 \\
1.29759 \\
1.45379 \\
1.65731 \\
1.97041\end{array}$ & $\begin{array}{l}0.80039 \\
0.88142 \\
0.97008 \\
1.06822 \\
1.17884 \\
1.30704 \\
1.46215 \\
1.66461 \\
1.97651\end{array}$ & $\begin{array}{l}0.82243 \\
0.90113 \\
0.98751 \\
1.08361 \\
1.19246 \\
1.31908 \\
1.47277 \\
1.67383 \\
1.98420\end{array}$ & $\begin{array}{l}0.84870 \\
0.92532 \\
1.00939 \\
1.10311 \\
1.20968 \\
1.33421 \\
1.48599 \\
1.68523 \\
1.99366\end{array}$ & $\begin{array}{l}0.87782 \\
0.95307 \\
1.03532 \\
1.12685 \\
1.23100 \\
1.35302 \\
1.50233 \\
1.69918 \\
2.00514\end{array}$ & $\begin{array}{l}0.90870 \\
0.98332 \\
1.06444 \\
1.15433 \\
1.25637 \\
1.37588 \\
1.52238 \\
1.71626 \\
2.01902\end{array}$ & \\
\hline $\begin{array}{l}.90 \\
.97 \\
.98 \\
.99\end{array}$ & $\begin{array}{l}2.05436 \\
2.17067 \\
2.32689 \\
2.57632\end{array}$ & $\begin{array}{l}2.17241 \\
2.32851 \\
2.5778\end{array}$ & $\begin{array}{l}2.05930 \\
2.17534 \\
2.33124 \\
2.58025\end{array}$ & $\begin{array}{l}2.06371 \\
2.17952 \\
2.33514 \\
2.58377\end{array}$ & $\begin{array}{l}2.06953 \\
2.18502 \\
2.34026 \\
2.58839\end{array}$ & $\begin{array}{l}2.07686 \\
2.19195 \\
2.34672 \\
2.59421\end{array}$ & & & & \\
\hline & .5 & .60 & .6 & .70 & .75 & .80 & .8 & W & .9 & 6. \\
\hline 05 & $\begin{array}{l}0.23810 \\
0.34210 \\
0.42601 \\
0.50060 \\
0.57012\end{array}$ & & & & & & & & & \\
\hline $\begin{array}{l}5 \\
60 \\
60 \\
7 \\
80 \\
80\end{array}$ & 2.05638 & 2.08130 & & & $\begin{array}{l}1.10361 \\
1.18366 \\
1.26875 \\
1.35090 \\
1.46309 \\
1.58010 \\
1.72059 \\
1.90335 \\
2.18580\end{array}$ & $\begin{array}{l}1.13 \\
1.21 \\
1.30 \\
1.39 \\
1.50 \\
1.62 \\
1.76 \\
1.94 \\
2.23\end{array}$ & & & & \\
\hline $\begin{array}{l}9 \\
98 \\
95\end{array}$ & $\begin{array}{l}55619 \\
06114 \\
+736 \\
+736\end{array}$ & 2 & & $\begin{array}{l}2.7 \\
2.7\end{array}$ & & & & & & \\
\hline
\end{tabular}


where $\rho, \theta$ are the usual polar coordinates stretched by a factor $\sigma_{x}$ and where

$$
0 \leqq \frac{\sigma_{y}}{\sigma_{x}}=c \leqq 1
$$

Simple transformations reduce $P(K, c)$ to

$$
P(K, c)=\frac{1}{\pi_{c}} \int_{0}^{\pi^{2} / 2} e^{-B w 0} \int_{0}^{\pi} e^{\Lambda \text { wocoso }} d \theta d w
$$

where

$$
A=\frac{1-c^{2}}{2 c^{2}} \text { and } B=\frac{1+c^{2}}{2 c^{2}} .
$$

The integral over $\theta$ in (4) is referred to in $[4,($ p. 46)], and may be expressed as

$$
\int_{0}^{\pi} e^{A u \cos \theta} d \theta=\pi I_{0}(A w)
$$

where $I_{0}(x)$ is defined by the following Taylor and asymptotic expansions re. spectively

$$
\begin{aligned}
& I_{0}(x)=\sum_{n=0}^{\infty}\left(\frac{1}{n !}\right)^{2}\left(\frac{x}{2}\right)^{2 n} \\
& I_{0}(x) \sim \frac{e^{x}}{\sqrt{2 \pi x}} \sum_{n=0}^{N^{\prime}} \frac{[(2 n) !]^{2}}{2^{5 n}(n !)^{3}} x^{-n} .
\end{aligned}
$$

The relations.(7) and (8) are given in [t] on pages 20 and 58 , respectively.

Two computation schemes were used for computing $P$. If $A K^{2} \leqq 40$ (an arbitrary choice), then the following recurrence relation was used to compute $P$ :

(9) $T_{2 n}=\frac{2 n-1}{2 n}\left(\frac{A}{B}\right)^{2} T_{2 n-2}-\frac{1}{B c}\left[\left(\frac{A K^{2}}{t}\right)^{2 n-1} e^{-B K^{2} / 2}\left\{\frac{A K^{2}}{t}+n\left(\frac{A}{B}\right)\right\}\right]\left(\frac{1}{n !}\right)^{2}$

where

$$
T_{0}=\frac{1}{B c}\left(1-e^{-B K^{2} / 2}\right)
$$

and

$$
P=\sum_{n=0}^{N} T_{2 n}
$$$$
A K^{-2} \leqq 40
$$

If $A K^{2}>40$ then the following recurrence relation was used to compute $P$ :

$$
M_{2 n+1}=\frac{1}{2 A c} \cdot \frac{1}{\sqrt{ } \pi} \cdot \frac{2}{2 n-1} \frac{[(2 n) !]^{2}}{2^{4 n}(n !)^{3}}\left(A K^{2}\right)^{-(2 n-1) / 2} e^{-K^{2 / 2}}
$$$$
-\frac{1}{2.1} \frac{2 n-1}{2 n} M_{2 n-1}
$$

where

$$
M_{1}=\frac{1}{\sqrt{1-c^{2}}} \cdot \frac{2}{\sqrt{\pi}} \int_{K / \sqrt{\mathrm{g}}}^{\infty} e^{-w^{2}} d w
$$


and

$$
P=1-\sum_{n=0}^{N^{\prime}} M_{2 n+1}
$$

Equation (9) is obtained by substituting (7) into (4), transforming the upper limit on the $W$ integration from $K^{2} / 2$ to $A K^{2} / 4$, interchanging summation and integration, and then performing two successive integrations by parts on the integral that occurs as part of the general $n$th term of the series. The upper limit, $N$, of the sum that appears in (11) is determined when

$$
\left|T_{2 N}\right| \leqq \epsilon\left|\sum_{n=0}^{N} T_{2 n}\right|
$$

where $\epsilon$ is chosen to the order of accuracy to which $P$ is desired.

The recurrence relation given by (12) is derived by substituting (8) into (4), interchanging summation and integration, and by considering the integral from $A K^{2}$ to infinity rather than from 0 to $A K^{2}$. Two integrations by parts on the integral that occurs as part of the general $n$th term of the series yield (12). The integer $N^{\prime}$ is determined such that

$$
\left|M_{2 N^{\prime}+1}\right| \leqq \epsilon\left|\sum_{n=0}^{N^{\prime}} M_{2 n+1}\right|
$$

The restriction of (12) to the region $A K^{2}>40$ insures at least eight-digit accuracy in $P$ before the terms $M_{2 n+1}$ eventually begin to increase in magnitude. The $\epsilon$ 's in (15) and (16) were set at $10^{-8}$.

Inasmuch as equal intervals in $P$ and $c$ were desired, a Newton-Raphson procedure was used to determine $K$ for a given $P$ and $c$; accordingly

$$
K_{n}=K_{n-1}-\frac{\frac{1}{c} \int_{0}^{\left(K_{n-1}^{2} / 2\right)} e^{-B w} I_{0}(A w) d w-P}{\frac{1}{c} K_{n-1} e^{-\left(B K_{n-1}^{2} / 2\right)} I_{0}\left(\frac{A K_{n-1}^{2}}{2}\right)}
$$

where $K_{n}$ represents the $n$th iterate for $K$.

The efficiency and accuracy of the computation are indicated by the fact that the average time required to evaluate a $K$ to eight significant digits for a given $P$ and $c$ was 50 milliseconds on NORC. The accuracy of the results was checked by evaluating the same $K$ by both (9) and (12) in the region of $A K^{2}=40$. Thirty terms were used for this purpose. This region is where both series (11) and (14) require the largest number of terms, and consequently where truncation and rounding errors should be the largest. Some further checks to insure eight-digit accuracy were obtained by evaluating some of the integrals by the direct application of Simpson's Rule. The entire table presented herein required less than 30 seconds of computing time on NORC.

5. Acknowledgment. The problem itself was presented to the Naval Weapons Laboratory, Dahlgren, Virginia, by the first author named. The second author carried out the actual solution of the problem as given in section 4 , above. Both authors 
wish to thank Mr. Irv Learman, Naval Weapons Laboratory, who did the programming and coding, and Mr. John Walker, Naval Weapons Laboratory, who programmed and coded the editing procedure for setting up the table.

Special Projects Office

Navy Department

Washington, District of Columbia

Naval Weapons Laboratory

Dahlgren, Virginia

1. Nat. Bur. Standards Appl. Math. Ser. No. 50, Tables of the Normal Bivariate Distribution Function, U. S. Government Printing Office, Washington, D. C., 1959.

2. Arthor Grad \& S. Herbert Solomon, "Distribution of quadratic forms and some applications," Ann. Math. Statist., v. 26, n. 3, 1955, p. 464-477.

3. M. G. Kendall, The Advanced Theory of Statistics, v. 11, third edition, Hafner Publishing Co., New York, 1951.

4. A. Grat, G. B. MAthews \& T. M. MAc Robert, A Treatise on Bessel Functions, Macmillan \& Co., London, 1091. 